\section{ORIGINAL RESEARCH}

J.R. Gaughen, Jr
P. Raghavan

M.E. Jensen

D. Hasan

A.N. Pfeffer

A.J. Evans

\title{
Utility of CT Angiography in the Identification and Characterization of Supraclinoid Internal Carotid Artery Blister Aneurysms
}

BACKGROUND AND PURPOSE: Blister aneurysms of the supraclinoid ICA represent a rare but potentially catastrophic cause of SAH, often presenting both diagnostic and therapeutic dilemmas. We explore the utility of CTA in the identification and characterization of ICA blister aneurysms.

MATERIALS AND METHODS: We performed a retrospective review of catheter cerebral angiograms obtained at our institution over a 12-month period for evaluation of SAH, identifying 6 cases of ICA blister aneurysms. All patients underwent CTA and DSA for evaluation of SAH. The reports from the CTA and DSA studies were reviewed to identify aneurysms correctly diagnosed prospectively. Retrospective review of the CTA and DSA images was also performed. Review of the interpretations and images was performed for any follow-up studies.

RESULTS: All 6 patients presented with SAH, diagnosed by head CT. All patients subsequently underwent CTA prior to DSA evaluation. All 6 aneurysms were identified prospectively on initial DSA imaging. Of the 6 blister aneurysms, 4 (67\%) were identified prospectively; and 5 (83\%), retrospectively on CTA. All 6 patients underwent endovascular treatment with stent placement. Four of the 6 aneurysms underwent follow-up CTA (range, 9-22 days), including the 2 aneurysms that had been unidentifiable preprocedurally. All 4 blister aneurysms were seen postprocedurally by DSA. Three of these 4 (75\%) residual aneurysms were detected by CTA (both prospectively and retrospectively).

CONCLUSIONS: In the presence of SAH and otherwise negative findings on CTA, a catheter cerebral angiogram should be performed to absolutely exclude an ICA blister aneurysm.

ABBREVIATIONS: CTA $=\mathrm{CT}$ angiography; DSA = digital subtraction angiography; ICA = internal carotid artery; MIP = maximum intensity projections; $\mathrm{MPR}=$ multiplanar reconstruction; $\mathrm{SAH}=$ subarachnoid hemorrhage

$\mathbf{C}^{\mathrm{T}}$

TA is being increasingly used in the diagnostic triage of SAH. ${ }^{1}$ Ruptured intracranial aneurysms represent a high percentage of SAH, and CTA has been shown to have a high sensitivity and specificity in detecting these ruptured aneurysms. ${ }^{2-4}$ However, with its temporal and higher spatial resolution, DSA remains the criterion standard for evaluating SAH. ${ }^{5}$

ICA blister aneurysms are rare wide-neck shallow aneurysms arising from nonbranching sites on the wall of the supraclinoid ICA. ${ }^{6}$ They differ both morphologically and histologically from saccular aneurysms and likely represent a distinct pathophysiology, appearing to more closely resemble pseudoaneurysms. ${ }^{7}$ As such, they are a potentially catastrophic cause of SAH, often presenting both diagnostic and therapeutic dilemmas. Their shallow profiles and broad bases make them difficult to detect with imaging.

In this case series, we demonstrate the imaging appearance of ICA blister aneurysms and explore the utility of CTA in the identification and characterization of these lesions.

\section{Received May 26, 2009; accepted after revision August 5.}

From the Department of Radiology (J.R.G., P.R., M.E.J., A.J.E.) and University of Virginia School of Medicine (A.N.P.), University of Virginia Health System, Charlottesville, Virginia; and Department of Neurosurgery, (D.H.), University of lowa Hospitals and Clinic, lowa City, lowa.

Please address correspondence to John R. Gaughen, Jr, MD, Department of Radiology, Box 800170, Charlottesville, VA 22908; e-mail: jrg4@virginia.edu

DOI 10.3174/ajnr.A1893

\section{Materials and Methods}

We performed a retrospective review of all catheter cerebral angiograms performed at our institution during an 8-month period (July 2007 through March 2008) for evaluation of SAH, identifying 6 cases diagnosed as ICA blister aneurysms. A total of 119 cerebral angiograms were obtained during that time period, identifying 89 ruptured aneurysms. ICA blister aneurysms were defined as shallow broadbased aneurysms arising from nonbranching sites of the supraclinoid ICA. These aneurysms have a characteristic triangular appearance and are clearly not associated with the supraclinoid ICA branch vessels. All patients underwent both CTA and DSA in the diagnostic evaluation of SAH. Postprocessing of CTA images included MPRs, MIPs, and volume-rendered 3D images.

Analysis of both prospective and retrospective data was performed. The official reports (reviewed and signed by the attending physician) from the CTA and DSA studies were reviewed to identify ICA blister aneurysms diagnosed prospectively by the interpreting physician. At our institution, CTA images are interpreted by the diagnostic neuroradiology staff, while DSA images are interpreted by the interventional neuroradiology staff. Retrospective review of the CTA and DSA images was also performed by 2 experienced interventional neuroradiologists (A.J.E., M.E.J.) and 1 experienced diagnostic neuroradiologist (P.R.). This was performed to compare these images with the prospective diagnoses, to determine whether diagnostic differences between CTA represented real findings or misinterpretation and to correlate the imaging characteristics of these aneurysms between the 2 modalities. Both prospective and retrospective interpretations of CTA data included evaluation of source, MPR, MIP, and volume-rendered 3D images. Review of the interpretations and im- 
ages was also performed for any follow-up studies, again to identify residual ICA blister aneurysms and to determine the correlation between the postprocedural CTA and DSA appearances. The physicians performing retrospective review were not blinded to the prospective findings.

\section{Results}

Six patients were included in this cohort, all diagnosed by DSA with ICA blister aneurysms. The cohort included 5 women and 1 man, with a mean of 50 years of age (range, 39-69 years of age).

All 6 patients presented with SAH, diagnosed by unenhanced head CT. The average Hunt and Hess grade at admission was 2.2 (range, 1-3), and the average Fisher grade was 3.2 (range, 1-4). All patients subsequently underwent diagnostic CTA before DSA evaluation. All 6 aneurysms were identified prospectively on initial DSA imaging. Of the 6 patients diagnosed with ICA blister aneurysms, $4(67 \%)$ were identified prospectively on CTA, with the other 2 (33\%) not identified prospectively by CTA (false-negatives). On retrospective review of the initial CTA images, 5 of the $6(83 \%)$ aneurysms were identified. One of the prospectively missed aneurysms on CTA was retrospectively seen, though partially obscured by a poor imaging technique. In this case, CTA image acquisition was inadvertently performed by using thicker sections; lowering the resolution of the source, MPR, and MIP images; and introducing step-off artifacts. The other missed aneurysm was not identifiable on CTA either prospectively or retrospectively. The initial CTA morphology of the 4 visible aneurysms closely correlated with the imaging characteristics demonstrated on DSA.

All 6 patients underwent endovascular treatment with stent placement, with an overlapping stent technique. Three of these patients subsequently underwent coil embolization, 1 of which subsequently needed parent vessel (ICA) coil occlusion for definitive treatment.

All 6 patients underwent follow-up DSA imaging (currently ranging from 6 to 12 months). However, CTA is not routinely used for long-term follow-up and is usually performed in the early posttreatment period for evaluation of other complications, such as vasospasm. Therefore, only 4 of the 6 aneurysms underwent follow-up CTA (range, 9-22 days), including both of the aneurysms that had been prospectively unidentifiable preprocedurally. All 4 of these blister aneurysms were seen postprocedurally by DSA. Three of these 4 (75\%) residual aneurysms were prospectively detected by CTA, while the fourth was not visible by CTA, either prospectively or retrospectively. The 2 patients who did not undergo follow-up CTA imaging showed no residual aneurysm on intermediate DSA imaging follow-up.

The 2 aneurysms undergoing CTA follow-up that were prospectively identified by CTA preprocedurally showed imaging characteristics that closely correlated with the DSA findings, with aneurysm patency demonstrated on both modalities. The aneurysm that had been retrospectively identified on initial CTA became clearly visible on the follow-up CTA, closely correlating with the morphology of the aneurysm on follow-up DSA. The other previously undetected aneurysm remained undetectable on the follow-up study, despite demonstrating patency on the correlative follow-up DSA.

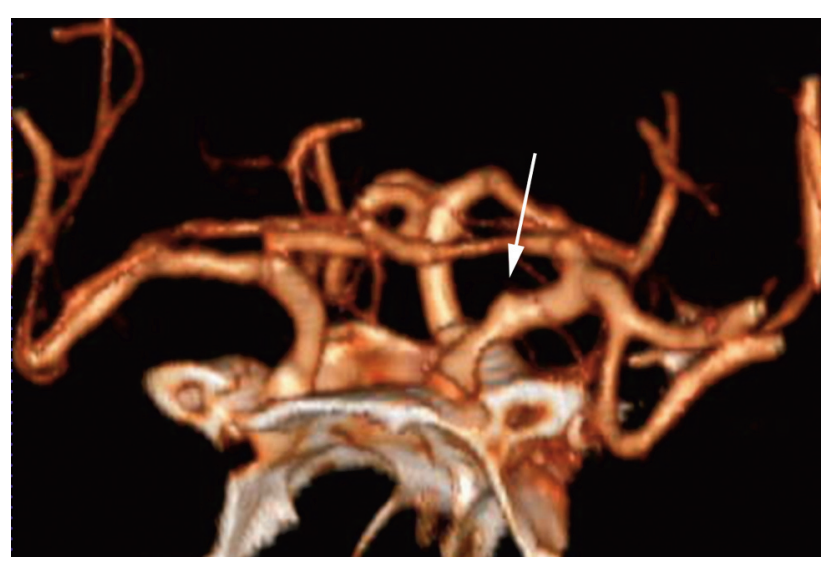

Fig 1. Left supraclinoid ICA blister aneurysm. Volume-rendered 3D CTA image shows the characteristic morphology of supraclinoid ICA blister aneurysms (white arrow): wide-neck shallow outpouching of the medial wall of the left supraclinoid ICA

\section{Discussion}

The term "blister aneurysm" has been used to describe aneurysms arising from nonbranching sites of the wall of the supraclinoid ICA. ${ }^{8}$ These lesions, with an estimated incidence of $0.9 \%-6.5 \%$ of ruptured intracranial aneurysms, ${ }^{6}$ represent a rare but potentially catastrophic cause of SAH, often presenting both diagnostic and therapeutic challenges. Compared with saccular aneurysms, these lesions tend to have a more precipitous clinical course, enlarging rapidly and rebleeding frequently. Morphologically, they appear as wide-neck shallow outpouchings of the anterior, medial, or lateral wall of the supraclinoid ICA (Fig 1). At surgery, they often contain a fragile thin wall and a broad communication with the parent vessel, without an identifiable neck. Histologically, these lesions have been shown to represent focal wall defects covered with thin fibrous tissue and adventitia, lacking the usual collagen layer, findings indicative of pseudoaneurysms. ${ }^{7}$

All lesions in our study arose from nonbranching sites of the supraclinoid ICA, evenly distributed between the anterior, medial, and lateral walls. Initial DSA imaging in all cases demonstrated a characteristic shallow triangular appearance with a broad-based attachment to the parent vessel. The 4 blister aneurysms that were diagnosed prospectively with CTA closely resembled their DSA counterparts (Fig 2), as did the 3 identified by CTA on short-term follow-up (Fig 3). Indeed, the subtlety of these aneurysms often represents a diagnostic challenge, both with CTA and DSA. As they evolve, these lesions often become more evident, enlarging and taking on a more saccular appearance (Fig 4).

Because of the broad-based shallow profiles of these aneurysms, in addition to an understanding of the morphology and distribution of these lesions, meticulous technique and a high index of suspicion are often both necessary to make this diagnosis.

Numerous studies have been published in the medical literature describing the sensitivity and specificity of CTA in the detection of intracranial aneurysms in the setting of SAH. Indeed, at our institution, we use this technology to triage patients with SAH, which helps define lesions and treatment and tailors informed consent for patients and their families. However, our results in this small cohort with blister aneurysms of 

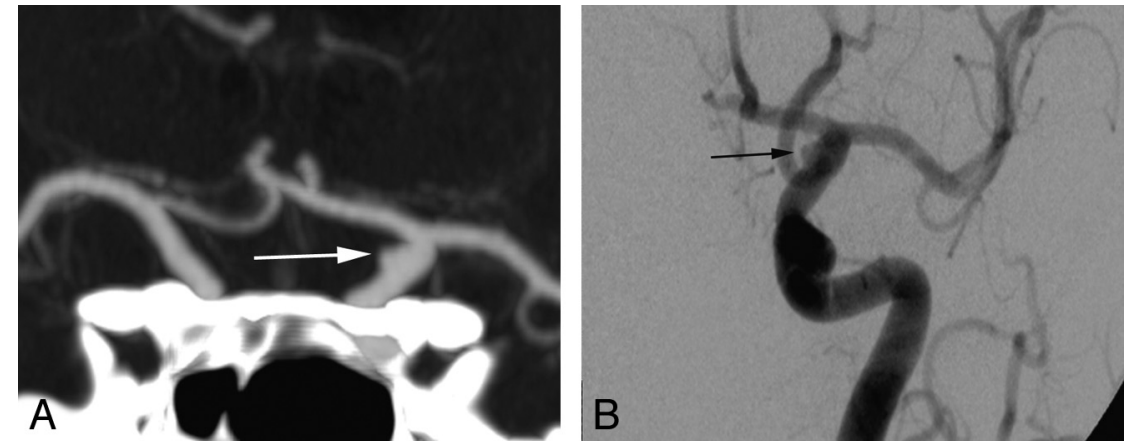

Fig 2. Left supraclinoid ICA blister aneurysm. Coronal MIP CTA image (A) shows the morphology of the blister aneurysm (white arrow), which closely resembles its DSA counterpart (black arrow, B).
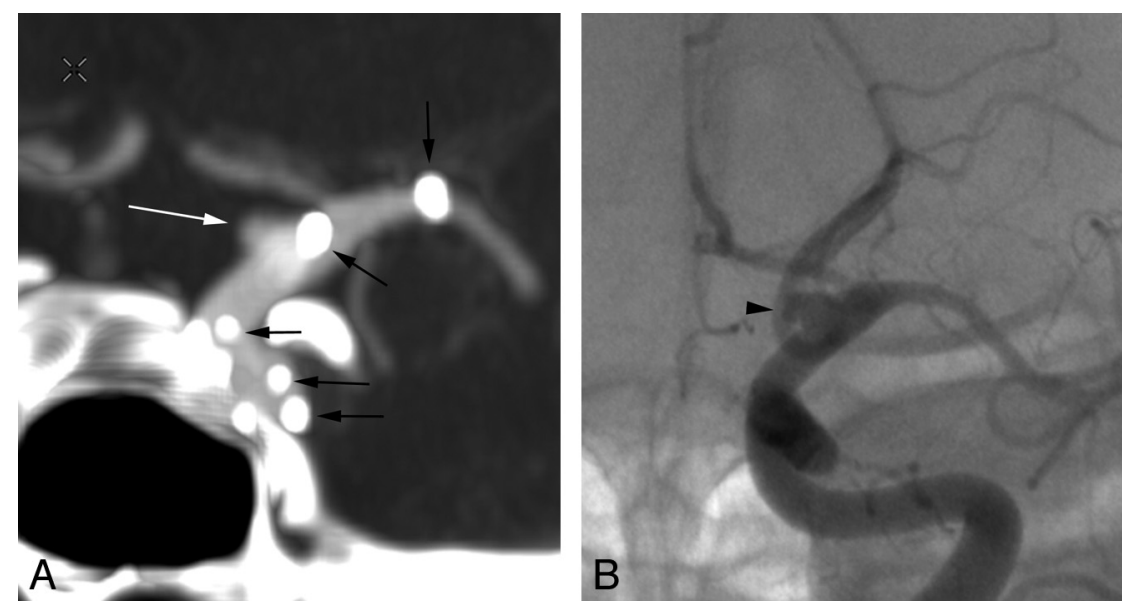

Fig 3. Left supraclinoid ICA blister aneurysm posttreatment. Coronal MIP CTA image (A) shows the morphology of the residual blister aneurysm (white arrow), which again resembles its DSA counterpart (black arrowhead, B), though the dome of the aneurysm appears slightly more prominent on the DSA study $(B)$. Note that the radiopaque tines of the overlapping stents used in the treatment are clearly seen on the CTA image (black arrows, A) but not on the DSA image.
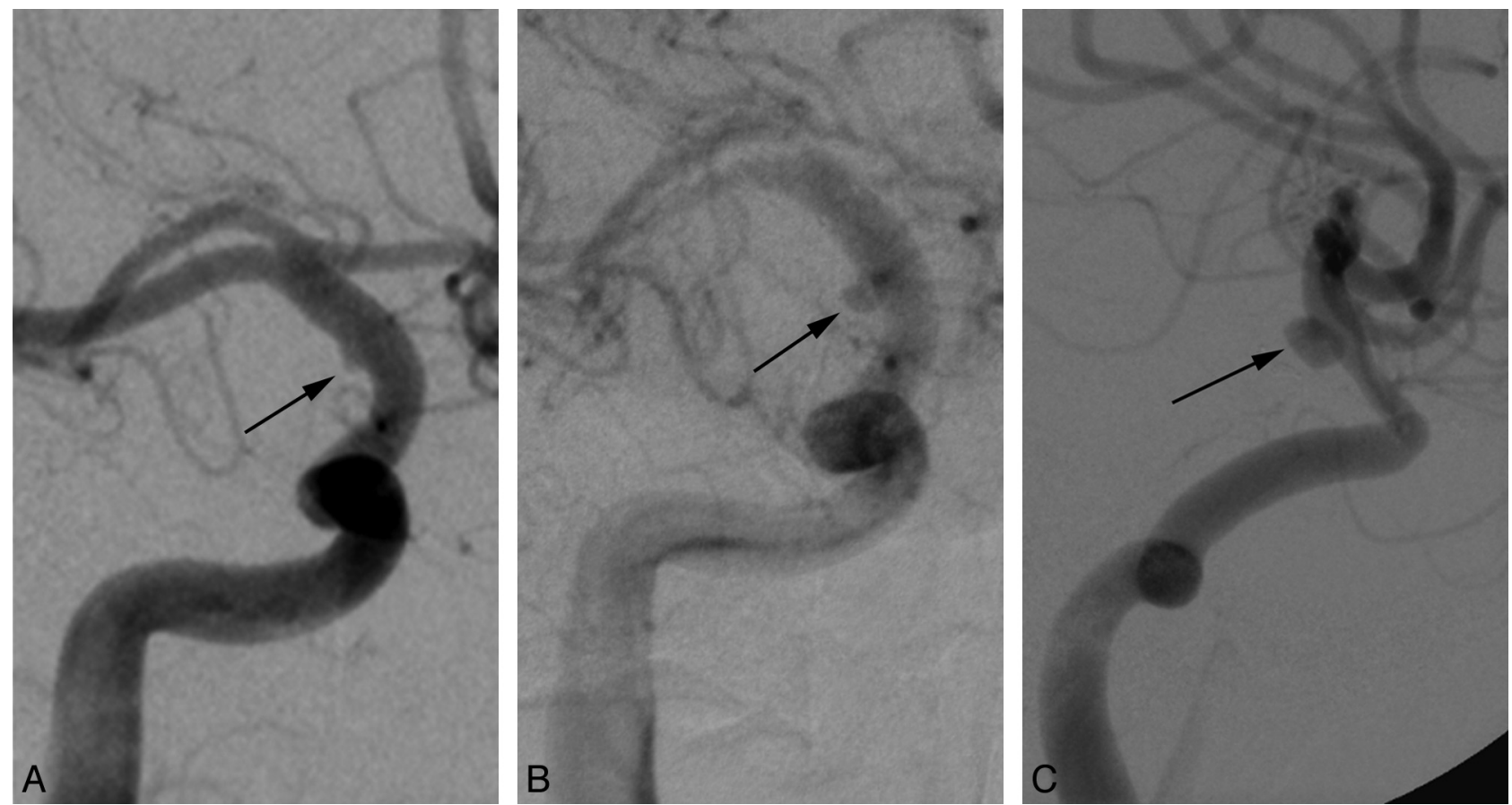

Fig 4. Right supraclinoid ICA blister aneurysm. At presentation, this blister aneurysm (black arrow, A) manifests as a subtle shallow outpouching, which becomes progressively larger and more saccular at 2-week $(B)$ and 3-month $(C)$ follow-ups, despite treatment with overlapping stents and subsequent coil embolization. 

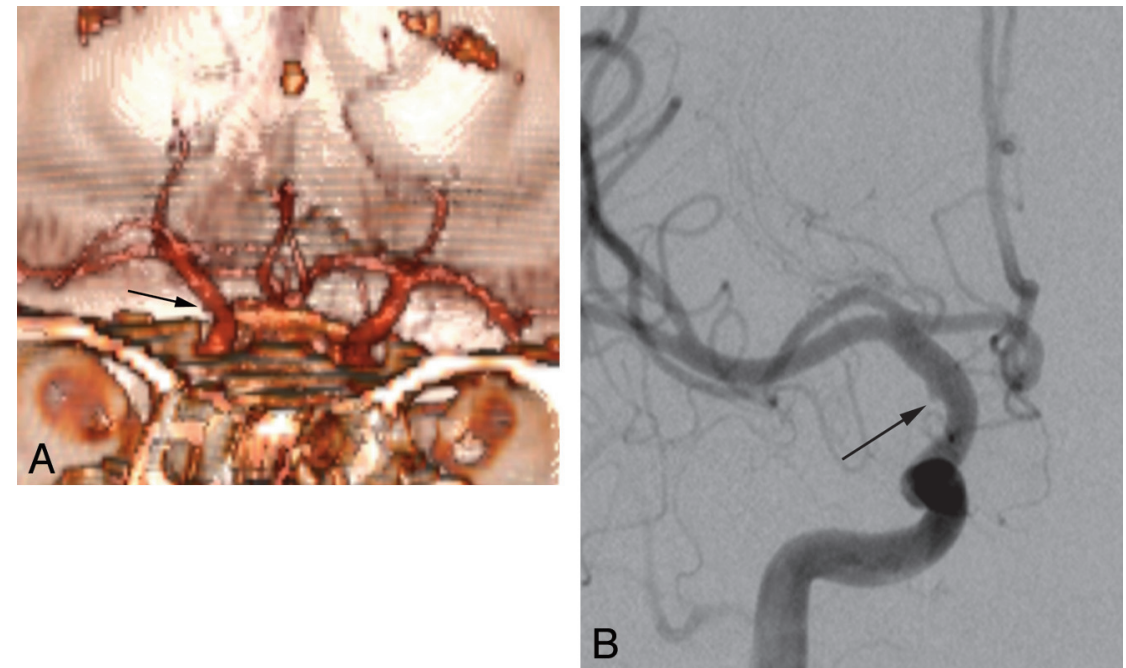

Fig 5. Right supraclinoid ICA blister aneurysm. This aneurysm (black arrow) was not identified prospectively but appears visible on retrospective review. A, Volume-rendered 3D CTA image shows a poor imaging technique, with a relatively low resolution and prominent step-off artifacts. $B$, The correlative DSA clearly delineates the small blister aneurysm (black arrow).
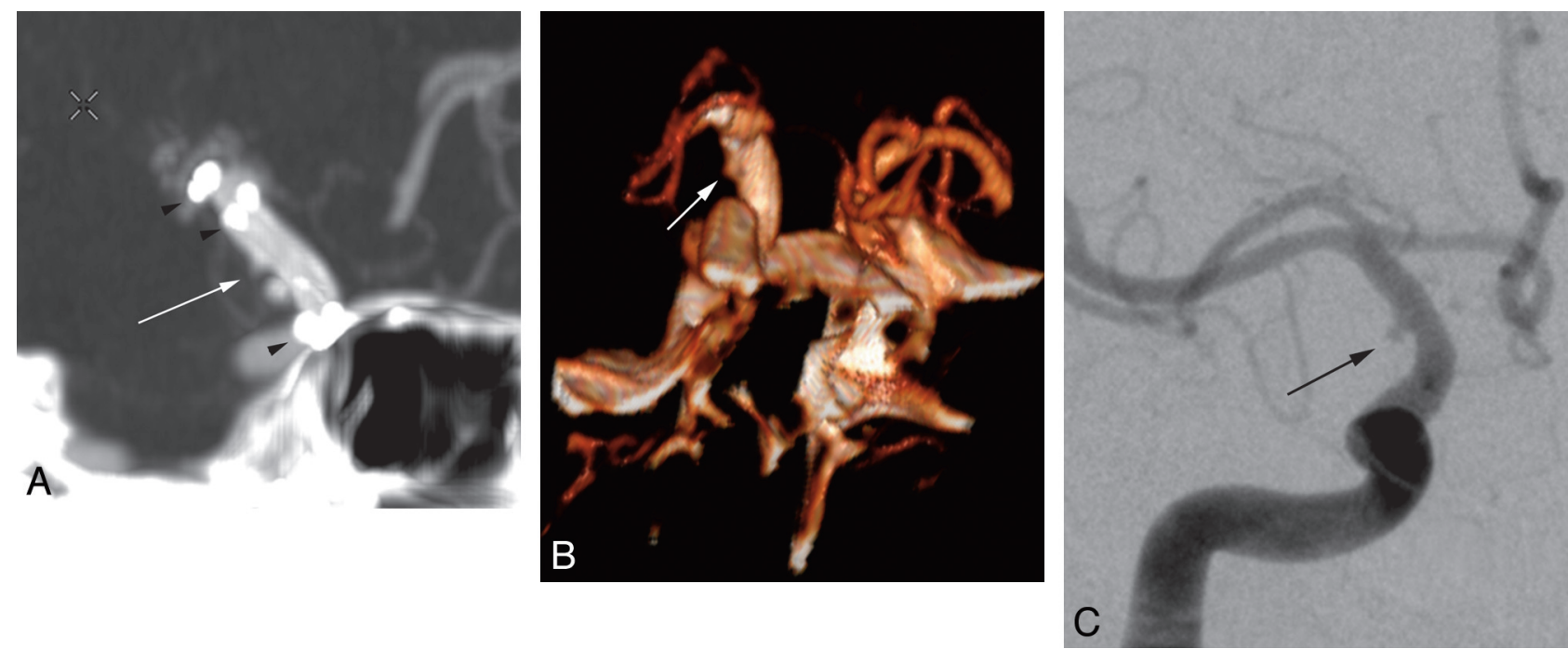

Fig 6. Right supraclinoid ICA blister aneurysm posttreatment. With adequate imaging and postprocessing parameters on the short-term CTA follow-up (following treatment of the blister aneurysm in Fig 5), the residual blister aneurysm (white arrow) is much more clearly identified ( $A$ and $B$ ) and closely resembled the appearance of the aneurysm on the correlative DSA (black arrow, C). Note the radiopaque tines of the overlapping stents used in the treatment identified on the CTA images (black arrowheads, A).

the supraclinoid ICA show that CTA may lack the sensitivity to effectively exclude the presence of this entity. This finding is not altogether surprising given the small size and shallow profiles of these aneurysms, because CTA lacks the spatial resolution of DSA imaging.

Our study illustrates the importance of optimizing CTA techniques in identifying these lesions. One of our CTA studies that produced a prospective false-negative result showed improper data acquisition and processing, resulting in suboptimal MPRs and 3D images. In this case, CTA image acquisition was inadvertently performed by using thicker sections; lowering the resolution of the source, MPR, and MIP images; and introducing step-off artifacts. In retrospect, this lesion was present on the CTA but was largely obscured due to the poor imaging technique (Fig 5). Our follow-up CTA in this patient, with its improved technique, which included decreased section thickness and increased pitch, clearly evinced the lesion (Fig 6). In our experience, other technical factors, such as im- proper bolus timing and venous contamination, have also been proved to obscure small aneurysms.

Our other false-negative CTA finding is more perplexing. Both the initial and follow-up CTA findings were negative, whereas both corresponding DSA studies showed relatively obvious abnormalities (Fig 7). The imaging and postprocessing techniques appear to have been optimized in both of these CTAs, but the abnormality remained invisible. Timing may have been a contributing factor, with a time delay of 3 days between each CTA and its corresponding DSA. Such a time lapse could certainly allow growth of the lesion, because we have noted that these blister aneurysms tend to grow rapidly. Spatial resolution probably did not contribute because lesions of a similar size were clearly visible in the 4 patients in whom the lesions were identified with CTA.

Our study is limited by its retrospective evaluation and small study size. Although our case series suggests that CTA is insufficiently sensitive to exclude the presence of ICA blister 

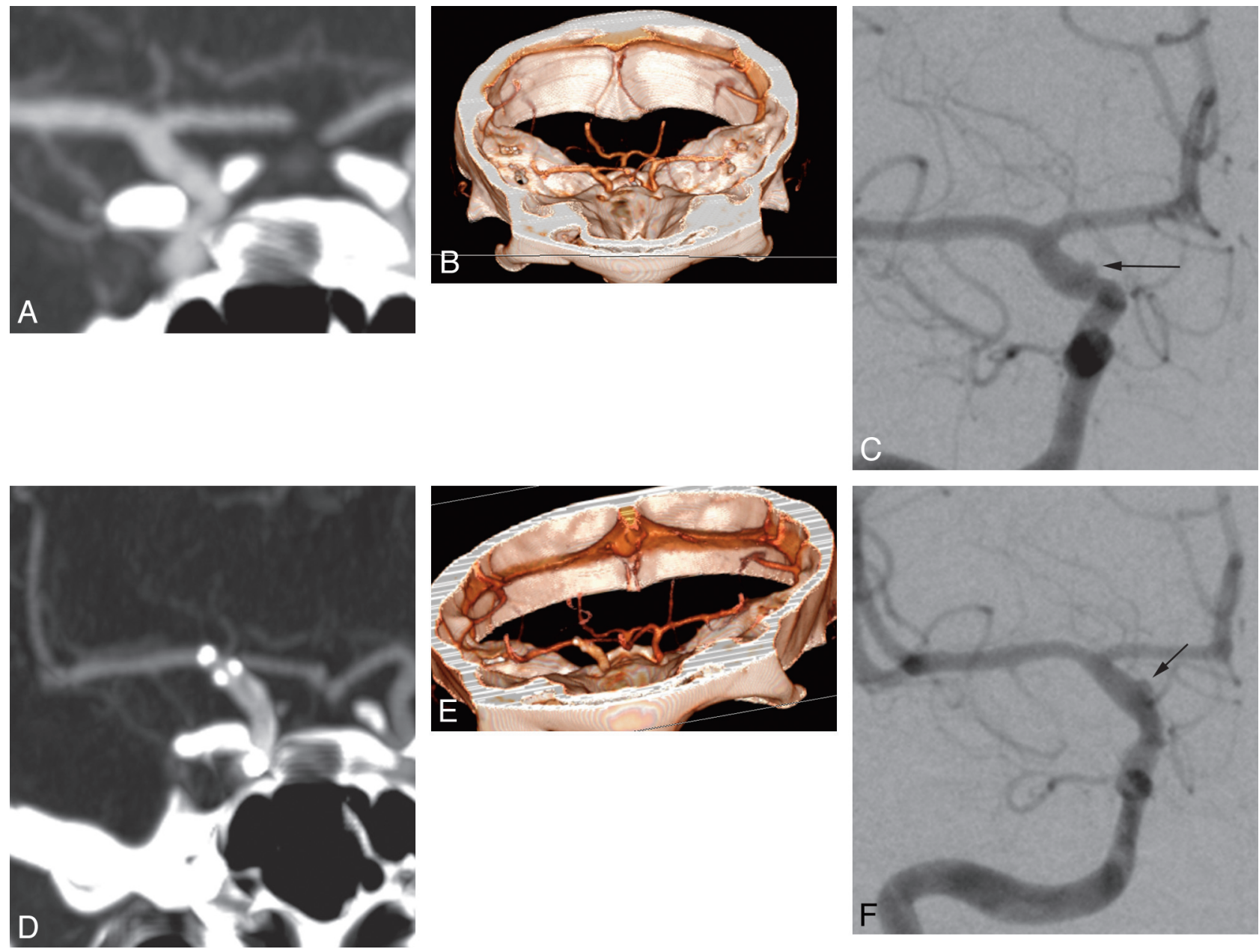

Fig 7. Right supraclinoid ICA blister aneurysm, pre- and posttreatment. The blister aneurysm is not detectable on initial CTA images ( $A$ and $B)$ but is clearly delineated on the correlative DSA image (black arrow, C). On the short-term posttreatment CTAs ( $D$ and $E$ ), the residual aneurysm remains invisible, while it is still clearly seen on the correlative posttreatment DSA image (black arrow, $\mathrm{F}$.

aneurysms, further investigation with a larger patient population and prospective evaluation would more clearly delineate these findings.

\section{Conclusions}

Blister aneurysms of the supraclinoid ICA remain an elusive diagnosis, particularly by using CTA. ICA blister aneurysm can be accurately diagnosed and characterized by CTA with a relatively high sensitivity, but not in $100 \%$ of cases. Thus, in the face of SAH and otherwise negative CTA findings, a catheter cerebral angiogram should be obtained to absolutely exclude a blister aneurysm.

\section{References}

1. Agid R, Lee SK, Willinsky RA, et al. Acute subarachnoid hemorrhage: using 64 slice multi-detector CT angiography to "triage" patients' treatment. Neuroradiology 2006;48:787-94. Epub 2006 Sep 29

2. Romijn M, Gratama van Andel H, van Walderveen MA, et al. Diagnostic accuracy of CT angiography with matched mask bone elimination for detection of intracranial aneurysms: comparison with digital subtraction angiography and 3D rotational angiography. AJNR Am J Neuroradiol 2008;29:134-39

3. Yoon DY, Lim KJ, Choi CS, et al. Detection and characterization of intracranial aneurysms with 16-channel multidetector row $\mathrm{CT}$ angiography: a prospective comparison of volume-rendered images and digital subtraction angiography. AJNR Am J Neuroradiol 2007;28:60-67

4. McKinney AM, Palmer CS, Truwit CL, et al. Detection of aneurysms by 64section multidetector $\mathrm{CT}$ angiography in patients acutely suspected of having an intracranial aneurysm and comparison with digital subtraction and 3D rotational angiography. AJNR Am J Neuroradiol 2008;29:594-602

5. van Rooij WJ, Sprengers ME, de Gast AN, et al. 3D rotational angiography: the new gold standard in the detection of additional intracranial aneurysms. AJNR Am J Neuroradiol 2008;29:976-79

6. Ogawa A, Susuki M, Ogasawara K. Aneurysms at no branching sites in the supraclinoid portion of the internal carotid artery: internal carotid artery trunk aneurysms. Neurosurgery 2000;47:578-86

7. Ishkawa T, Nakamura N, Houkin Ket al. Pathological consideration of a "blister" aneurysm at the superior wall of the internal carotid artery: case report. Neurosurgery 1997;40:403-06

8. Abe M, Tabuchi K, Yokoyama H, et al. Blood blister like aneurysms of the internal carotid artery. J Neurosurg 1998;89:419-24 